\title{
Photonic Characteristics of Langmuir-Blodgett Self-Assembled Monolayers of Colloidal Silica Particles
}

\author{
Edgar-Julián Cabrera*, Lina M. Jaller, Roger Amade, Sabine M. Portal ${ }^{\dagger}$, \\ Esther Pascual, and Enric Bertran \\ FEMAN Group, IN ${ }^{2} U B$, Department of Applied Physics and Optics, \\ Universitat de Barcelona, C/Martí i Franquès, 1, 08028, Barcelona, Spain
}

\begin{abstract}
Monodispersed colloidal crystals based on silica sub-micrometric particles were synthesized using the Stöber-Fink-Bohn process. The control of nucleation and coalescence result in improved characteristics such as high sphericity and very low size dispersion. The resulting silica particles show characteristics suitable for self-assembling across large areas of closely-packed 2D crystal monolayers by an accurate Langmuir-Blodgett deposition process on glass, fused silica and silicon substrates. Due to their special optical properties, colloidal films have potential applications in fields including photonics, electronics, electro-optics, medicine (detectors and sensors), membrane filters and surface devices. The deposited monolayers of silica particles were characterized by means of FESEM, AFM and optical transmittance measurements in order to analyze their specific properties and characteristics. We propose a theoretical calculation for the photonic band gaps in 2D systems using an extrapolation of the photonic behavior of the crystal from 3D to $2 D$. In this work we show that the methodology used and the conditions in self-assembly processes are decisive for producing high-quality two-dimensional colloidal crystals by the Langmuir-Blodgett technique.
\end{abstract}

Keywords: Colloidal Crystals, Langmuir-Blodgett (LB), Monolayer, Nanoparticles, Self-Assembly.

Structures produced at the nanometer and sub-micrometer scale have been studied in recent years due to the outstanding physical, mechanical, chemical, and optical properties obtained at these scales. ${ }^{1,2}$ Colloidal crystals can be used in the manufacture of high-quality templates, with remarkable dispersion, order and size, and thin membranes. These properties make them suitable for photonics and smart surfaces with controllable photo-switched wettability properties. ${ }^{3,4}$ They are also promising for applications in bioengineering and photovoltaic cells ${ }^{5}$ and for template patterning. ${ }^{6}$

Monodisperse silica particles were prepared using the Stöber-Fink-Bohn method. ${ }^{7}$ In a solution of ethanol and tetraethylorthosilicate (TEOS), ammonia was added to fix the $\mathrm{pH}$ to basic. After some minutes, ultrapure water was added to promote hydrolysis. The entire synthesis process occurred under vigorous stirring. At the end of the process, the silica particle surface was functionalized with 3-aminopropyltrimethoxysilane (APTS) to give the final particles the required hydrophilic properties. ${ }^{8}$

\footnotetext{
* Author to whom correspondence should be addressed.

'Present address: Department of Photonics and Terahertztechnology, Ruhr-University Bochum, 44780 Bochum, Germany.
}

The process was then repeated using different chemical concentrations, stirring speed, time of addition of chemicals and reaction time. We found that at stirring speeds higher than $450 \mathrm{rpm}$, the resulting silica particles are not spherical and are highly variable in size and shape. The opposite occurs when a low stirring speed in a range of 450 to $250 \mathrm{rpm}$ is used, when the resulting particles are monodisperse in size and shape. ${ }^{9}$ Something similar occurs with the addition time of the chemicals during the synthesis process; with fast addition of the chemicals, many agglomerates form and particles shapes are not well defined. However, when the chemicals are added dropwise, agglomerates are negligible and the particles are spherical. These results may be attributed to the time required in the nucleation process for the final particle size and moreover, to the increment in particle interaction due to a stirring velocity that allows small particles to nucleate, ultimately leading to a larger size distribution. We can say that the particle size, shape and dispersity depend largely on the stirring speed of the sample during the process and on the time of addition and reaction of the chemicals. The size of the silica particles obtained ranged from 200 to $500 \mathrm{~nm}$ for different solutions. These results were reproducible several times following the same synthesis 
Table I. Detailed growth conditions used in the synthesis of silica particles. The stirring speed and time of chemical addition play an important role in the final particle size and shape. These values were used to synthesize particles $500 \mathrm{~nm}, 360 \mathrm{~nm}$ and $200 \mathrm{~nm}$ in diameter.

\begin{tabular}{lcccc}
\hline \multicolumn{5}{c}{ Parameters for the synthesis of $\mathrm{SiO}_{2}$ particles } \\
\hline $\begin{array}{l}\text { Sample } \\
\text { order }\end{array}$ & $\begin{array}{c}\text { Stirring } \\
\text { speed (rpm) }\end{array}$ & $\begin{array}{c}\text { Chemical addition } \\
\text { time (s) }\end{array}$ & $\begin{array}{c}\text { Diameter } \\
(\mathrm{nm})\end{array}$ & $\begin{array}{c}\text { Dispersion } \\
(\%)\end{array}$ \\
\hline 1 & 300 & 5 & 500 & 3 \\
2 & 260 & 5 & 522 & 5 \\
3 & 320 & 8 & 360 & 6 \\
4 & 340 & 4 & 200 & 4 \\
\hline
\end{tabular}

procedure. Table I shows the parameters and the particle size obtained in this study.

The Langmuir Blodgett (LB) technique was used to deposit the resulting silica spheres, due to the relative simplicity with which the conditions of self-assembly can be achieved and the accurate control of the deposition process achieved even over large areas. ${ }^{10,11}$

The sub-micrometric particles were dispersed in the gas-liquid interphase (air-water interphase) of the LB system using a microsyringe. At first, the particles are disordered, then they are compressed using barriers until they forms a uniform ordered monolayer; at which point we start the process of transferring the layer onto a substrate. Monitoring the surface pressure and surface area, (the $\Pi-A$ isotherm) we control the deposition process to achieve the compact phase. The colloidal crystals were deposited on glass, fused silica and silicon substrates $\left(25-100 \mathrm{~cm}^{2}\right)$ in one deposition step using a large trough LB system.

The monolayer uniformity and the compactness of the particles depend on the parameters used during the LB deposition before and after layer transfer to the solid substrate. ${ }^{12,13}$ In our experiments, we used a compression velocity of $8-10 \mathrm{~mm} / \mathrm{min}$ after the spreading procedure of the solution, and a timeout of 20 minutes was required to allow the solvent to evaporate and the particles to relax. The monolayers were transferred during the upstroke at a fixed rate of $1 \mathrm{~mm} / \mathrm{min}$. All the experiments were performed at a constant temperature of $25.0{ }^{\circ} \mathrm{C} \pm 0.2{ }^{\circ} \mathrm{C}$.

The surface morphology and structure of the monolayers were determined by images obtained using a Field Emission Scanning Electron Microscope (FESEM, Hitachi 4100 ) with a resolution of $5 \mathrm{~nm}$, an accelerating voltage of $15 \mathrm{kV}$ and an emission current of $10 \mu \mathrm{A}$. We also used an Atomic Force Microscope (AFM, XE-70) from Park Systems in non-contact mode. The resulting films consisted of a single monolayer of sub-micron particles arranged in compact hexagonal structured domains (Figs. 1 and 2). Figure 2 shows an example of an FESEM image of the particle monolayer. The crystalline defects that can be observed limited the monocrystalline domains. Fourier analysis of the image shows the order of the structure and the main symmetry axes. In some cases, the monolayers

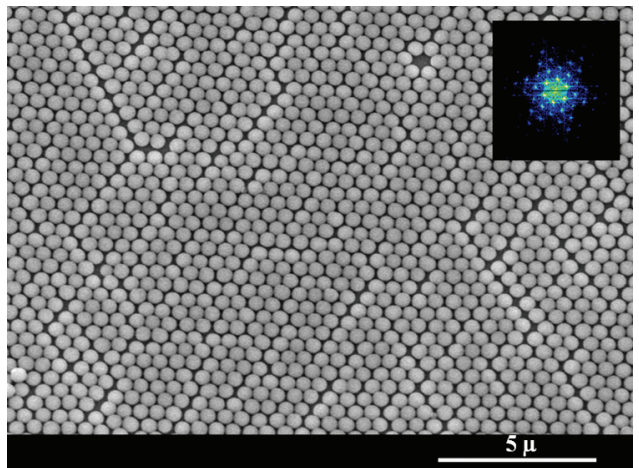

Fig. 1. FESEM image of particle monolayer, where domains, order and particle structure are observed. Almost all the domains are oriented in the (111) direction plane of the substrate, and only a few are oriented in the (100) direction plane. The fast Fourier transform (FFT) of the picture is presented in the inset.

show large perfect monocrystalline 2D domains, with more than $2 \times 10^{4}$ particles of $500 \mathrm{~nm}$ diameter. In almost cases, the domains appear ordered in a hexagonal compact structure corresponding to the [111] direction normal to the substrate. $^{14}$

Using the FESEM images, we determined the average particle diameter and the circularity of the deposited monolayers using the free distribution Image ${ }^{\circledR}$ software. ${ }^{15}$ Applying a series of masks to determine the threshold contrast of the deposited monolayers of particles on the substrate in different samples, we obtained diameters of $200 \pm$ $15 \mathrm{~nm}, 360 \pm 10 \mathrm{~nm}$ and $500 \pm 10 \mathrm{~nm}$, and circularity near to $0.952,0.938$ and 0.902 , respectively. The data obtained for the particle diameters were represented using statistical software and plotting the measured diameter against the number of particles counted. For example, in the sample whose particle diameter was determined to be approximately $500 \mathrm{~nm}$, we obtained a size dispersion of lower than 3\%; demonstrating we had obtained a monodispersed sample, with a mean particle size of $510.46 \mathrm{~nm}$ (Fig. 3).

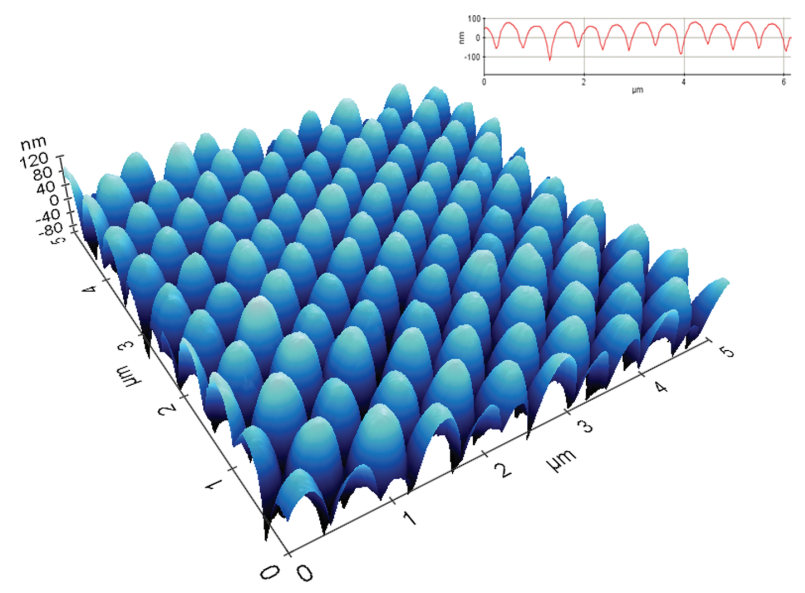

Fig. 2. AFM image of the silica particles. The image shows the uniformity in the morphology of the monolayer over the substrate and the compact hexagonal structured domains. Top inset: surface profile.

Nanosci. Nanotechnol. Lett. 4, 1-5, 2012 


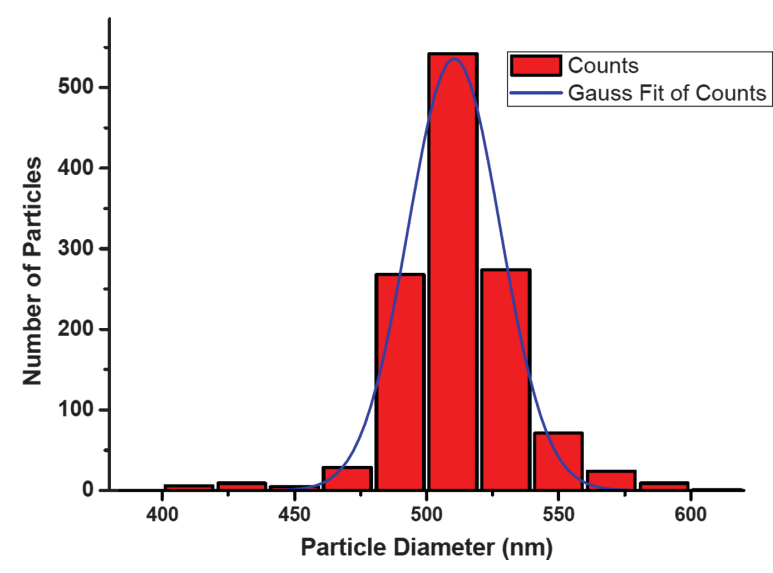

Fig. 3. Histogram with a Gaussian function of the particle size distribution of $\mathrm{SiO}_{2}$ particles on a silicon substrate. Mean particle size, $D_{m}=$ 510.46 and the standard deviation, $\sigma=17.45$.

Figure 4 shows the Fourier analysis of the FESEM images of $\mathrm{SiO}_{2}$ particles of $500 \mathrm{~nm}$ in diameter. In this analysis, the periodicity of the hexagonal symmetry of the monolayer for large areas can be observed. Here we compare the contrast between the bright spots (order and directionality) and periodic rings (loss of directionality). Figure 4(a) shows a highly-ordered hexagonal geometric distribution for an area of $4 \mu \mathrm{m} \times 4 \mu \mathrm{m}$ that shows only bright spots. Something similar can be observed in Figures 4(b) and (c), whose areas correspond to $8 \mu \mathrm{m} \times$ $8 \mu \mathrm{m}$ and $16 \mu \mathrm{m} \times 16 \mu \mathrm{m}$, respectively; but some very faint rings appear that indicate a small loss of directionality. Figure 4(d) $(32 \mu \mathrm{m} \times 32 \mu \mathrm{m})$ shows clear rings and diffused bright spots. At this size, the directionality is lost but the order is preserved at short distances. The bright spots are missing in Figure 4(e) $(64 \mu \mathrm{m} \times 64 \mu \mathrm{m})$ indicating an area several times larger than a monocrystalline domain. In our experiments, the photonic monocrystalline domains were between 16 - and $32-\mu \mathrm{m}$-sized regions. The large size of these regions is due to low particle size dispersion and accurate control of the technological parameters (low transfer velocity, maximum surface pressure, constant temperature and high fluidity of the particles in the Langmuir monolayer) which are needed to control the LB deposition. ${ }^{16}$ Defects such as dislocations appear due to vibrations and variations in the velocity or surface pressure. Sporadic defects such as vacancies are related to particle size singularities.

Transmittance measurements were taken using a Lambda 950 UV/Vis spectrophotometer from Perkin Elmer. We used a beam light incident normal to the substrate in a wavelength range of 200-2500 nm. Figure 5 shows the transmittance measurements corresponding to ordered particle monolayers deposited on fused silica substrates. The spectra shown correspond to a naked fused silica substrate and a series of samples of $360 \pm 10 \mathrm{~nm}$ and $500 \pm 10 \mathrm{~nm}$ silica particle diameters (Fig. 5).

The spectrum of the substrate shows no absorption from 2500 to $275 \mathrm{~nm}$. The spectra of the series of particles show photonic behavior that is evidenced by an electromagnetic coupling effect at a wavelengths of $\lambda_{\min }=587.6 \mathrm{~nm}$ and $\lambda_{\text {min }}=721.6 \mathrm{~nm}$. This effect is consistent with the position of the pseudogap in the (111) direction of the photonic band structure (Fig. 6) for $a / \lambda=0.9$ (Table I), where $a$ is the lattice parameter of the cubic cell and $\lambda$ is the photon
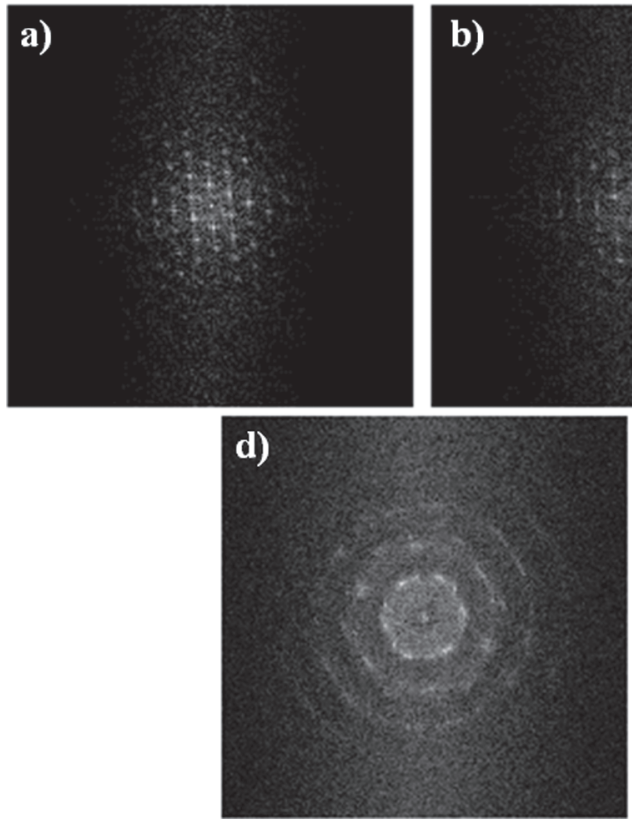
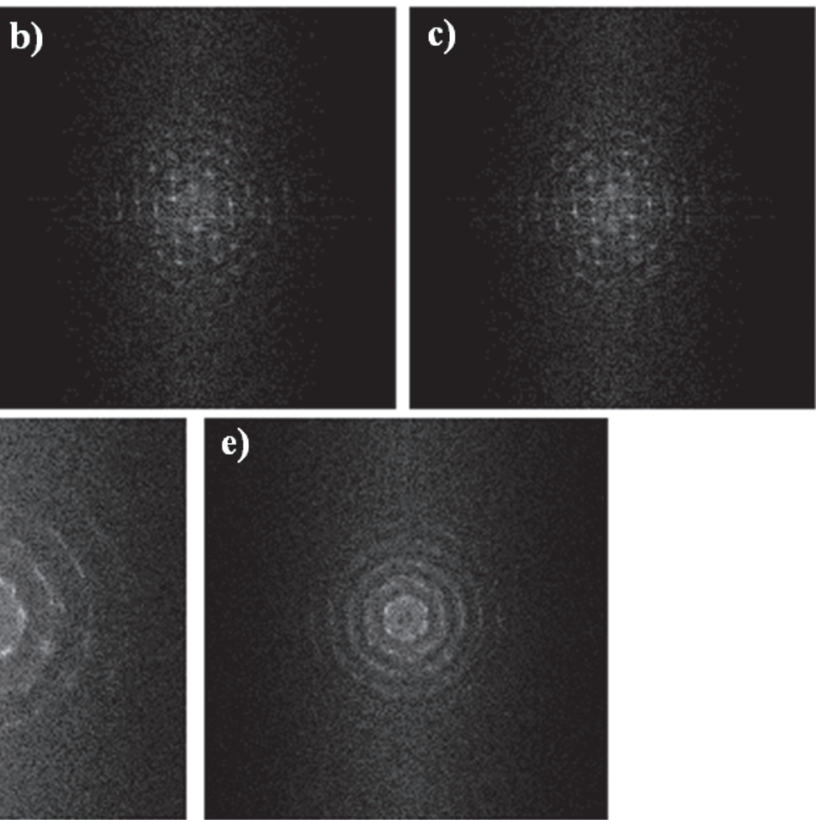

Fig. 4. The FFT of the FESEM images of silica particles $500 \mathrm{~nm}$ in diameter. Depending on the order of the bright spots, we can deduce the degree of periodicity of the hexagonal arrangement in the domains. The areas analyzed were (a) $4 \mu \mathrm{m} \times 4 \mu \mathrm{m},(\mathrm{b}) 8 \mu \mathrm{m} \times 8 \mu \mathrm{m},(\mathrm{c}) 16 \mu \mathrm{m} \times 16 \mu \mathrm{m}$, (d) $32 \mu \mathrm{m} \times 32 \mu \mathrm{m}$ and (e) $64 \mu \mathrm{m} \times 64 \mu \mathrm{m}$. 


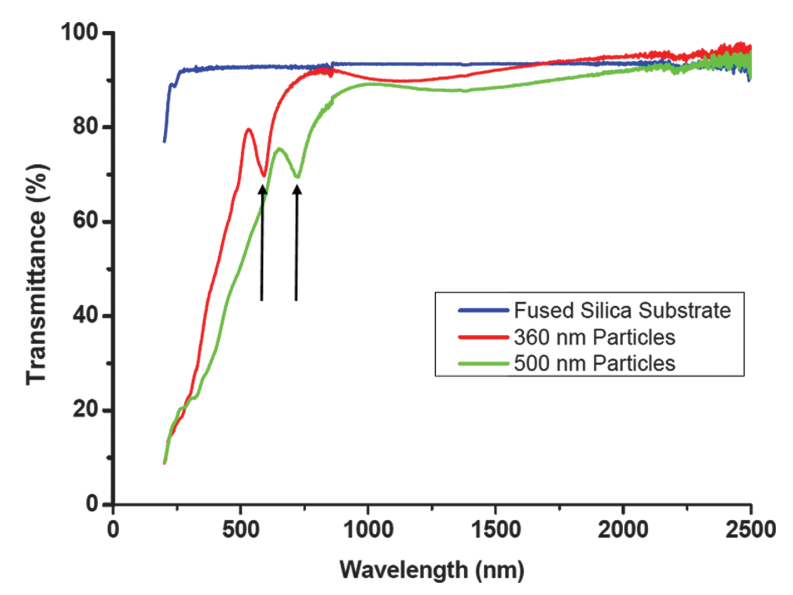

Fig. 5. Transmittance measurements on fused silica substrate (blue) and monodispersed silica particles on fused silica. The arrows correspond to the minimum values of transmittance at $\lambda_{\min }=587.6 \mathrm{~nm}$ and $\lambda_{\min }=$ $721.6 \mathrm{~nm}$ for particles $360 \mathrm{~nm}$ in diameter (red) and silica particles $500 \mathrm{~nm}$ in diameter (green), respectively.

wavelength. ${ }^{17}$ This photonic band structure corresponds to the fcc structure formed by ordered silica spheres in air. For this calculation we have taken the refractive index of silica spheres to be 1.42 and the volume fraction of a hexagonal compact monolayer to be $f=\sqrt{2 / 3}$. So the effective $2 \mathrm{D}$ radius $\left(R_{2 D}\right)$ of the particles becomes:

$$
R_{2 D}=\sqrt[6]{\frac{2}{3}} \cdot R
$$

where $R$ is the particle radius.

In Table II the values of the particle diameters obtained from the FESEM image analysis are compared with the values calculated for the photonic behavior (Figs. 5 and 6) and show good agreement.

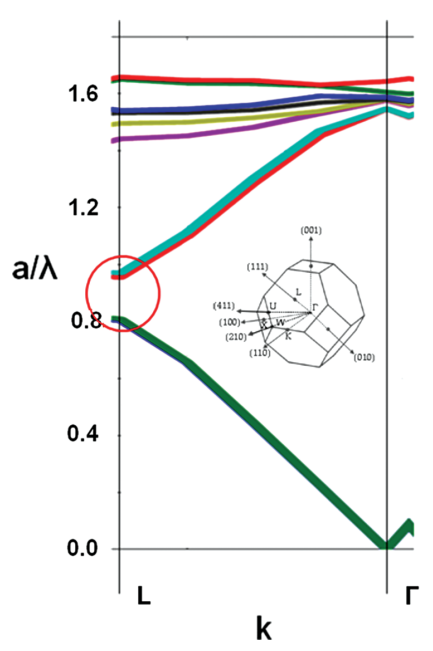

Fig. 6. Photonic band structure for an fcc structure of a 3D photonic crystal of spherical silica particles in an air matrix. The red circle indicates the pseudogap in the $L$ direction (111). Inset: the first Brillouin zone of the fcc structure with the correspondences between the reciprocal space and real space.
Table II. The table shows the particle diameters obtained using image analysis of the FESEM of silica particles deposited on fused silica substrates. The calculated theoretical values of the diameter using information related to the photonic behavior of the samples are in a good agreement with the calculated value for the pseudogap of the photonic band.

Particle diameters by FESEM image analysis and diameters calculated by the photonic behavior

\begin{tabular}{lcccc}
\hline $\begin{array}{l}\text { 2D photonic } \\
\text { crystal }\end{array}$ & $\lambda_{\text {min }}(\mathrm{nm})$ & $\mathrm{D}(\mathrm{nm})$ & $a / \lambda$ & $D_{\text {ph }}(\mathrm{nm})$ \\
\hline Sample 1 & 587.6 & $360 \pm 10$ & $0.90 \pm 0.08$ & $374 \pm 30$ \\
Sample 2 & 721.6 & $500 \pm 10$ & $0.90 \pm 0.08$ & $459 \pm 40$ \\
\hline
\end{tabular}

In short, silica sub-micrometric particles of different diameters were synthesized using the Stöber-Fink-Bohn method (sol-gel process) with a high monodispersity in size and shape; highly desirable characteristics for the manufacture of 2D photonic crystals. The silica particles were self-assembled in a closely-packed hexagonal 2D structure using the Langmuir-Blodgett technology. The monocrystalline domains obtained show dimensions of between 16 and $32 \mu \mathrm{m}$. In addition, these 2D photonic structures show a pseudogap for normalized light frequency $a / \lambda=0.9$ in the direction (111) of the real space (normal to the substrate).

The photonic properties of silica particle samples depend of the size of the particles and their morphology. Their photonic characteristics strongly depend on the size of their monocrystalline domains. In contrast, the 2D photonic characteristics were extrapolated from a 3D model assuming an fcc structure for the silica spheres. The results are in good agreement between the particle diameters determined by FESEM and those calculated from their photonic band structure applied to the transmittance measurements.

Acknowledgments: This work was financed by the research project NANOTRAPPING (CTQ2009-14674C02-01). The authors thank the Centres Cientifics $i$ Tecnologics of the University of Barcelona (CCiTUB) for the FESEM measurement facilities. We also thank the Solar Energy Group at the University of Barcelona for the transmittance measurements. Edgar-Julián Cabrera thanks the FPU scholarship program of the Spanish Ministerio de Educación, Cultura y Deporte for helpful funding during this work. The authors thank Dr. Shangping Guo for help with the calculation of the photonic band structure.

\section{References and Notes}

1. Y. Masuda, T. Itoh, and K. Koumoto, Langmuir 2, 4478 (2005).

2. T. Tlusty and S. A. Safran, Phil. Trans. R. Soc. Lond. A 359, 879 (2001).

3. L. Gao and T. J. McCarthy, Langmuir 23, 3762 (2007).

4. S. M. Abrarov, Sh. U. Yuldashev, T. W. Kim, S. B. Lee, H. Y. Kwon, and T. W. Kang, J. Lumin. 114, 86 (2005).

Nanosci. Nanotechnol. Lett. 4, 1-5, 2012 
5. G. Yu, J. Wang, H. Ishikawa, M. Umeno, T. Soga, T. Egawa, J. Watanabe, and T. Jimbo, Appl. Phys. Lett. 70, 3209 (1997).

6. M. Li, H. Schnablegger, and S. Mann, Nature 402, 393 (1999).

7. W. Stöber, A. Fink, and E. Bohn, J. Colloid Interface Sci. 26, 62 (1968).

8. S. Portal, M. Rubio-Roy, C. Corbella, M. A. Vallvé, J. Ignes-Mullol, and E. Bertran, Thin Solid Films 518, 1543 (2009).

9. S. Kalele, R. Dey, N. Hebalkar, J. Urban, and S. W. Gosav, Indian Academy of Sciences 65, 787 (2005).

10. Y. Huang and J. E. Pemberton, Colloids and Surfaces A: Physicochem. Eng. Aspects 360, 175 (2010).

11. H. Yang and P. Jiang, Langmuir 26, 13173 (2010).
12. S. Portal, M. Rubio-Roy, C. Corbella, M. A. Vallvé, J. Ignes-Mullol, and E. Bertran, Thin Solid Films 517, 1053 (2008).

13. M. A. Vallvé, Sistemes Nanoestructurats Mitjançant Monocapes de Langmuir, Ph.D. Thesis, Universitat de Barcelona (2006).

14. T. Kohoutek, J. Orava, T. Sawada, and H. Fudouzi, J. Colloid Interface Sci. 353, 454 (2011).

15. T. Ferreira and W. Rasband, ImageJ User Guide Developed at the National Institutes of Health, EUA (2011).

16. Y. L. Lee, Z. C. Du, and W. X. Lin, J. Colloid Interface Sci. 296, 223 (2006).

17. M. Ibisate, Cristales Fotónicos Basados en Ópalos, Ph.D. Thesis, Universidad Autónoma de Madrid (2003).

Received: $\mathrm{xx}$ Xxxx xxxx. Accepted: $\mathrm{xx}$ Xxxx xxxx. 